\title{
Enhancing Charge Transfer and Photoelectric Characteristics for Organic Solar Cells
}

\author{
Xiaofei Wang, ${ }^{1}$ Weiwei Pei, ${ }^{2}$ and Yuanzuo Li ${ }^{1}{ }^{1}$ \\ ${ }^{1}$ College of Science, Northeast Forestry University, Harbin, 150040 Heilongjiang, China \\ ${ }^{2}$ College of Science, Jiamusi University, 154001 Heilongjiang, China \\ Correspondence should be addressed to Yuanzuo Li; yzli@nefu.edu.cn
}

Received 2 July 2019; Revised 19 September 2019; Accepted 11 October 2019; Published 26 February 2020

Guest Editor: Scott L. Wallen

Copyright (C) 2020 Xiaofei Wang et al. This is an open access article distributed under the Creative Commons Attribution License, which permits unrestricted use, distribution, and reproduction in any medium, provided the original work is properly cited.

\begin{abstract}
The main purpose of this work is to analyze the effect of steric hindrance on the photoelectric performance of three different donor sensitizers (ZHG5, ZHG6, and ZHG7) by molecular theory simulation engineering. Photoelectric physical and photoelectric chemical parameters are investigated by means of frontier molecular orbital, global reactivity descriptors, optical absorption properties, fluorescent lifetime, charge density difference, and influence of external electric field. The results showed that the performance of the quinoxaline sensitizer was deteriorated by gradually increasing the steric hindrance to auxiliary donors. The optical properties of the hybridization of cir-coronene graphene quantum dot (GR) with the three dyes have been revealed, and the results show that graphene quantum dots can indeed improve the optical properties of solar cells. In addition, nine new molecules were designed by inserting six functional groups; it is found that inserting - $\mathrm{CN}$ in the acceptor part of the molecular structure is beneficial to the performance of the sensitizer.
\end{abstract}

\section{Introduction}

Energy is the driving force for the development of human society. However, due to the depletion of fossil energy, human society is facing an energy crisis. Therefore, the development and utilization of clean energy are particularly important. Solar cells have many advantages, such as clean and pollution-free, low cost, and renewable and have gradually become a hot research object. Since the advent of dyesensitized solar cells (DSSCs) in 1991, high-performance sensitizers have been a hot topic among researchers [1]. The common dye-sensitized solar cells are mainly composed of five parts: transparent conductive optical (TCO) glass, transparent nanocrystalline semiconductor thin films (photoanode), photoactive layer (dye and electrolyte solution without photoanode), redox electrolyte, and counter electrode (photocathode), which forms the sandwich's structure $[2,3]$. As a core component of dye-sensitized solar energy, the photoactive layer of different components directly affects the performance of the cells. As the filling material of the photoactive layer, the sensitizer can be divided into two types: metal sensitizer and nonmetal sensitizer. Nonmetallic sensitizer and polymers have the advantages of nonpollution, stability and well performance, which can be used as a potential candidate in the field of optoelectronics $[4,5]$.

The diversity of sensitizers has always been a research hotspot in the field of DSSCs, and the performance of sensitizers represented by D- $\pi$-A type structure is particularly outstanding, which has been unanimously recognized by the academic community [6-8]. Xu et al. [9] synthesized a new sensitizer with $\mathrm{D}-\pi-\mathrm{A}$ structure and applied it to the modified photoanode and found that the photoanode with 3D inverse opal nanostructure was more favorable to the photoelectric properties of DSSC. Huang and his collaborators [10] synthesized two novel $\pi$-conjugated moieties that could be part of $\pi$-bridge for the construction of D- $\pi$-A-configured photosensitizers for DSSC applications. The results demonstrated that lateral $\pi$-conjugated expansion can improve device performance better than the linear $\pi$-conjugated extension. Duerto and his collaborators [11] synthesized four new sensitizers for DSSCs with a tert-butyldimethylsilylether in order to test their physical 
and photovoltaic properties. The experimental results verify that the performance of the device is greatly improved when thiophene is inserted into the $\pi$-conjugated bridge part.

Many achievements have been made in the research on the performance of sensitizers in experiments, and the theoretical part has been widely used with the improvement of the density functional theory (DFT) technology [12-16]. In addition, graphene quantum dots have also been studied theoretically and experimentally as fillers of photosensitive layers $[17,18]$. Dong et al. [19] used DFT to investigate the tentative mechanism of the intramolecular electron and hole separation process at the quantum chemical level. The results implied that electrons are injected from QDs@GR nanomaterials into the cir-coronene graphene quantum dot and transition along with graphene sheet through $\pi^{*}$ orbitals to achieve interfacial separation of electron-hole. Gao and coworkers [20] used theoretical and experimental methods to simulate the optical properties of four sensitizers and circoronene graphene quantum dot nanocomposites. The results demonstrate that the optical properties of all the nanocomposites have enhanced compared with those of isolated sensitizers. Li et al. [21] used the method of quantum chemistry calculation to simulate a series of sensitizers with rigid fused $\pi$-conjugated bridge. The calculated results indicate that reducing the aromaticity of the $\pi$-conjugated bridge can promote intramolecular electron delocalization and enhance the electronic injection at the $\mathrm{Dye} / \mathrm{TiO}_{2}$ interface.

In this work, based on the research results of Chen and his collaborators [22], we used the method of quantum chemistry to further analyze how the steric hindrance affects the performance of the sensitizer (ZHG5, ZHG6, and ZHG7). In addition, sensitizers ZHG6 and ZHG7 were synthesized by adding auxiliary donors based on the molecular structure of ZHG5. The photophysical and photochemical characteristics of three sensitizers in tetrahydrofuran (THF) were simulated. Meanwhile, the optical properties of cir-coronene graphene quantum dot (we use GR to indicate the cir-coronene graphene quantum dot mentioned below) hybridization with ZHG5, ZHG6, and ZHG7 have been revealed. Subsequently, nine new molecules based on the molecular structure of ZHG5 have been designed to study the optical characteristics of sensitizers and the interface electron injection from the photoactive layer to semiconductors.

\section{Computational Details}

The DFT $[23,24]$ and TD-DFT [25-27] were used to analyze the ground-state and excited-state performance of the sensitizers. Moreover, the optimized geometries and frontier molecular orbitals of dyes were simulated by using B3LYP/6$3 \lg (\mathrm{d})[28-30]$. In order to make the theoretical simulation results more convincing, the functional used in the excited state needs to be screened. Based on sensitizer ZHG5, the UV-Vis absorption spectra simulated by six functionals (Cam-B3LYP, $\omega$ B97X, PBEPBE, MPW1PW91, LC- $\omega$ PBE, and B3LYP [31-36]) were compared with the experimental spectra. Functional Cam-B3LYP, whose performance was closest to the experimental results, was selected for subsequent calculation of the properties of excited states, and the results of the simulation are summarized in Table S1. Meanwhile, the excited-state properties of all sensitizers were calculated in THF solution by means of the conductor polarizable continuous model (C-PCM) [37].

In addition, adiabatic electron-transfer time $\left(\tau_{2}\right)$ and rate $\left(k_{\mathrm{ET}}\right)$ from sensitizers to semiconductor were simulated in THF solution. The electron-transfer time could be calculated by the following formula $[38,39]$ :

$$
\begin{aligned}
\hbar \Gamma & =\sum_{\mathrm{i}} p_{i}\left|\varepsilon_{i}-E_{\mathrm{LUMO}}\right|, \\
\tau(\mathrm{fs}) & =\frac{658}{\hbar \Gamma}(\mathrm{meV}),
\end{aligned}
$$

where $\hbar \Gamma$ is the broadening width, $p_{i}$ and $\varepsilon_{i}$ represent the adsorbate part of $i$ th molecular orbital for the sensitizer and its corresponding molecular orbital energy, and $E_{\mathrm{LUMO}}$ is the sum of the energies of the adsorbate in the unoccupied orbitals. All the theoretical results were obtained by Gaussian 09 [40].

\section{Results and Discussion}

3.1. Spatial Structures. The geometry structures of three investigated sensitizers ZHG5, ZHG6, and ZHG7 were optimized in tetrahydrofuran (THF) solution without symmetry constraint, and their spatial structures are available in Figure 1 and Scheme 1. With the donor part based on ZHG5, a benzene ring was inserted into the auxiliary donor portion of ZHG5 as a secondary electron-donating group, so we get sensitizer ZHG6. Based on the structure of ZHG6, a 2-(4-ethylhexyloxy)naphthalene $\left(D_{2}{ }^{*}\right)$ as the steric hindrance was inserted into the auxiliary donor group and we get molecule ZHG7. Some dihedral angles for all the optimized structures are summarized in Table 1 . The interfacial angles $\left(\theta_{1}\right)$ between the donor and auxiliary donor for three dyes are $-34.03^{\circ},-34.23^{\circ}$, and $33.28^{\circ}$, respectively. The twisted angles $\left(\theta_{2}\right)$ between the donor section and the $\pi$-conjugation part for three dyes are $43.88^{\circ}, 43.83^{\circ}$, and $39.77^{\circ}$, respectively, which means that the juncture section is distorted. Combining previous research in this area, we conclude that the donor portion of the dye molecule has a distorted structure; it inhibits the intermolecular $\pi-\pi$ aggregation on the semiconductor films. The $\pi$-spacers show good coplanarity with the acceptor unit with the dihedral angles $\left(\theta_{3}\right)$ less than $1.8^{\circ}$ in the three dyes. Thus, the $\pi$-bridges make excellent $\pi$-conjugation with the acceptor part, which facilitates for ICT and red shift of absorbance range. The dihedral angle $\theta_{4}$ between two benzene rings is unique to dye ZHG7, and its value is $82.05^{\circ}$. This approximately right-angled two $D_{2}$ planar structure indicates the existence of a steric hindrance effect by donor T-type stacking interaction, which results in the distortion of two structures that should be coplanar.

3.2. Frontier Molecular Orbital (FMO) Analysis. The molecular orbitals' transition ability can be reflected by the orbitals' energy levels. The calculated energy levels are shown in Table 2 and Figure 2. In addition, FMOs and charge density difference (CDD) of ZHG5, ZHG6, and ZHG7 are presented 

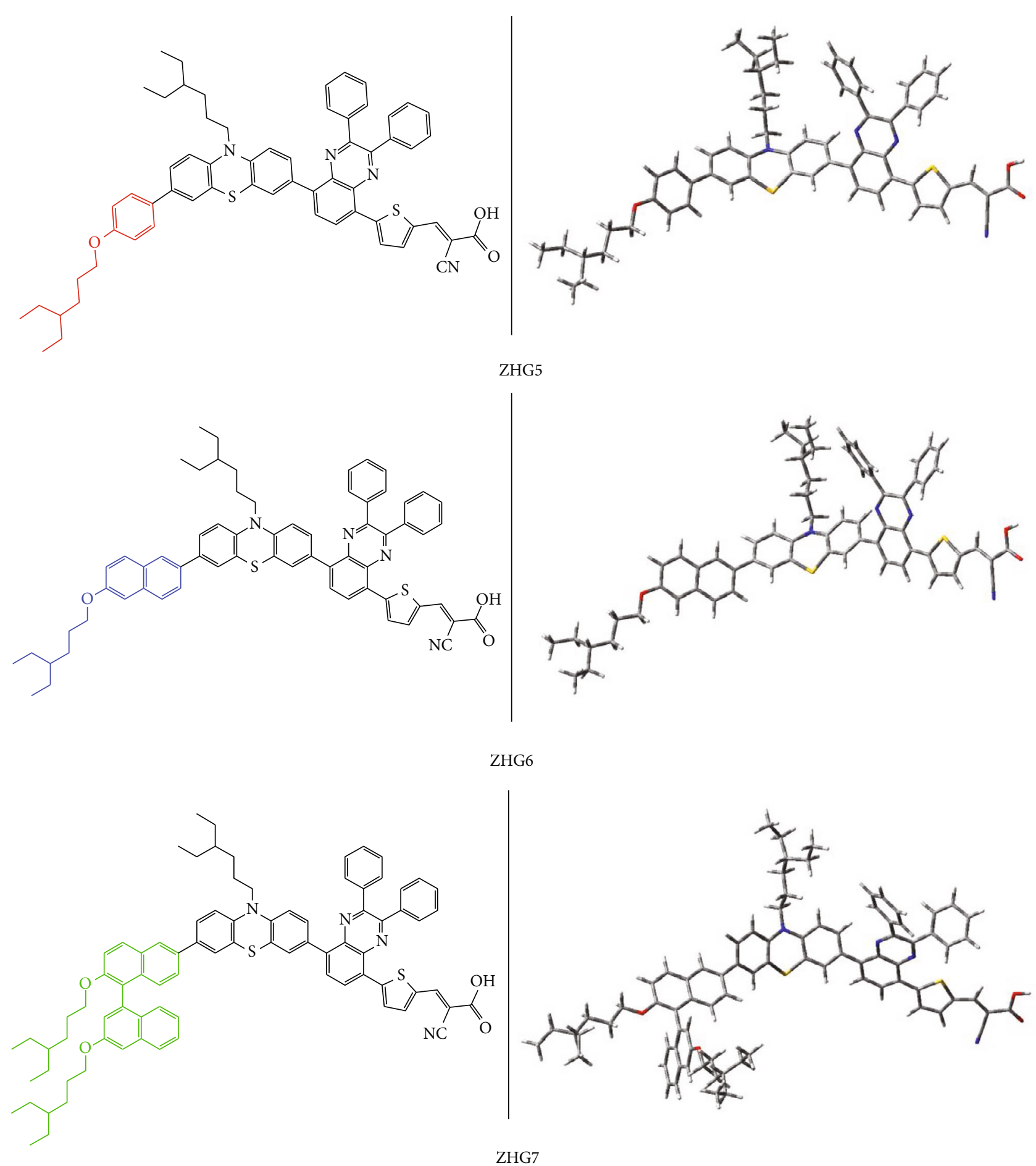

FIgURE 1: Chemical structures of ZHG5, ZHG6, and ZHG6.

in Figure 3. The energy levels of sensitizers need to meet the levels of $\mathrm{I}^{-} / \mathrm{I}_{3}{ }^{-}$couple and $\mathrm{TiO}_{2}$ conducting band edge. LUMO levels of the sensitizer are laying above the conduction band of the titanium dioxide $(-4.0 \mathrm{eV})$ [41], demonstrating that electrons can be transferred easily from the excited-state sensitizer to semiconductor. The HOMO levels of the sensitizer are under the redox potential of $\mathrm{I}_{3}^{-} / \mathrm{I}^{-}$electrolyte $(-4.6 \mathrm{eV})$ [42], which indicates that the electron-deprived sensitizers can quickly replenish the lost electrons from the electrolyte.
For ZHG5, the electron densities at HOMO are mainly occupied in the donor and auxiliary parts (see Figure 3). The electron densities aggregate the acceptor section at LUMO. The CDD can directly reflect the difference of electron density between the ground state and the first excited state. In Figure 3, the green (red) sections represent the negative (positive) electron density. As sensitizer ZHG5 is stimulated by light, electrons are transferred from the donor section to the acceptor moiety. As the acceptor group of the sensitizer 


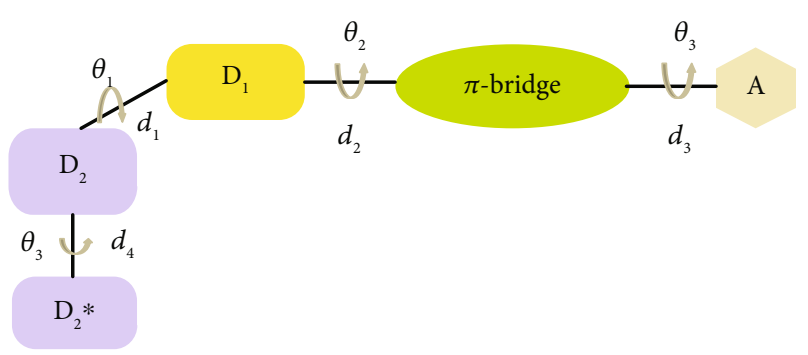

SCHEMe 1: Brief structure of D-D- $\pi-A$ dyes $\left(\theta_{1}-\theta_{4}\right.$ represent the dihedral angle; $d_{1}-d_{4}$ represent the bond length; $D_{2}{ }^{*}$ represents the 2-(4-ethylhexyloxy)naphthalene).

TABLE 1: Selected bond lengths and dihedral angles based on the optimized geometries of the three dyes in THF solution.

\begin{tabular}{cccc}
\hline & ZHG5 & ZHG6 & ZHG7 \\
\hline \multicolumn{4}{c}{ Bond length $(\AA)$} \\
$d_{1}$ & 1.483 & 1.483 & 1.482 \\
$d_{2}$ & 1.478 & 1.478 & 1.478 \\
$d_{3}$ & 1.425 & 1.424 & 1.425 \\
$d_{4}$ & -- & -- & 1.498 \\
\hline \multicolumn{4}{c}{ Dihedral angle $\left(^{\circ}\right)$} \\
$\theta_{1}$ & -34.03 & -34.23 & 33.28 \\
$\theta_{2}$ & 43.88 & 43.83 & 39.77 \\
$\theta_{3}$ & 1.73 & 1.54 & 1.36 \\
$\theta_{4}$ & -- & -- & 82.05 \\
\hline
\end{tabular}

TABLE 2: Energy levels and energy gaps of all the investigated dyes and its complexes in solvent $(\mathrm{eV})$.

\begin{tabular}{lccc}
\hline & $H$ & $L$ & $\Delta_{\mathrm{H}-\mathrm{L}}$ \\
\hline ZHG5 & -4.94 & -2.89 & 2.05 \\
ZHG6 & -4.95 & -2.89 & 2.06 \\
ZHG7 & -4.96 & -2.89 & 2.07 \\
\hline ZHG5/GR & -4.94 & -2.97 & 1.97 \\
ZHG6/GR & -4.96 & -2.97 & 1.99 \\
ZHG7/GR & -4.96 & -2.97 & 1.99 \\
\hline
\end{tabular}

is easily adsorbed on the semiconductor substrate, the electron transfer from the photosensitive layer to the photoanode is facilitated. The electron distributions of ZHG6 at the HOMO and LUMO are similar to those of ZHG5. As sensitizer ZHG7 is stimulated by light, intramolecular charge transfer occurs.

Table 2 shows that HOMO energy levels in investigated sensitizers is ZHG5 > ZHG6 > ZHG7, and the order of energy gaps is ZHG7 $>$ ZHG6 $>$ ZHG5, which indicates that the insertion of the benzene ring and 2-(4-ethylhexyloxy)naphthalene as steric hindrance caused the HOMO level of the photosensitizer to decrease, and it is not conducive to absorption spectrum bathochromic shift. The insertion of these functional groups into molecules leads to an increase in the energy gap, which is not conducive to photoexcitation.

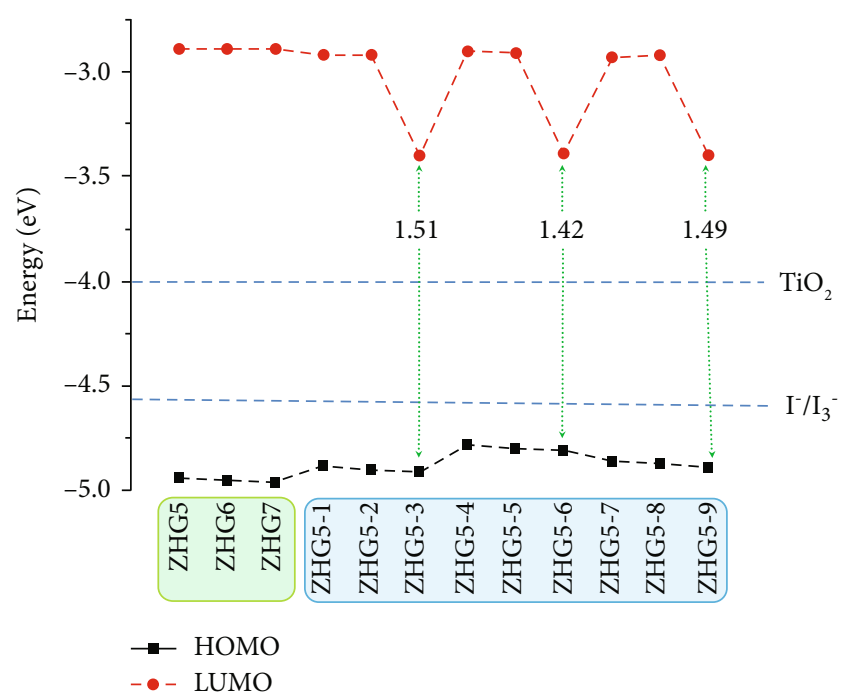

FIgURE 2: Molecular orbital energies and energy gaps of the molecules.

This may be the reason for the degradation of the overall performance of sensitizers, so the simulation results are in line with the experimental conclusions.

3.3. Global Reactivity Descriptors. On the basis of Koopmans' theorem [43-45], different global reactivity descriptors, i.e., electronegativity $(\chi)$, hardness $(\eta)$, softness $(S)$, chemical potential $(\mu)$ and electrophilicity index $(\Delta \mathrm{E})$, are computed by means of the energies of ionization potential (IP) and electron affinity (EA). The smaller ionization potentials are favorable for electron transfer to semiconductor substrate, and the larger electron affinities inhibit electron transfer from photo-anode to photosensitive layer. The electron affinities of the three sensitizers had no obvious changes. From Table 3, the ionization potentials are $4.813 \mathrm{eV}, 4.826 \mathrm{eV}$, and $4.827 \mathrm{eV}$ for ZHG5, ZHG6, and ZHG7, which is indicating that sensitizer ZHG5 is more efficient at injecting electrons into the semiconductor substrate.

Moreover, the total molecular energies $\left(E_{\text {TOTAL }}\right)$, chemical hardness $(\eta)$, chemical softness $(S)$, and electrophilicity index $(\Delta \mathrm{E})$ of the three dyes in a solvent phase were calculated. As shown in Table 3, chemical hardness is a measure of electron transfer resistance, and the results show that ZHG5 is $3.316 \mathrm{eV}$, which is the lowest value relative to ZHG6 $(3.328 \mathrm{eV})$ and ZHG7 $(3.330 \mathrm{eV})$. Chemical potential can reflect the ability of photosensitive molecules to transfer electrons to an equilibrium state after photoexcitation, it can be seen from Table 3 that the escaping ability of ZHG5 is strongest. The electrophilicity index is the property of a molecule to obtain electrons from the environment or to provide electrons to the environment. All three molecules have a negative electrophilicity index, which means all three dyes have the ability to provide electrons to the environment.

3.4. Optical Characteristics. The UV-Visible absorption spectra were simulated via TD-DFT in THF solvent. The wavelength of the maximum molar extinction coefficient 


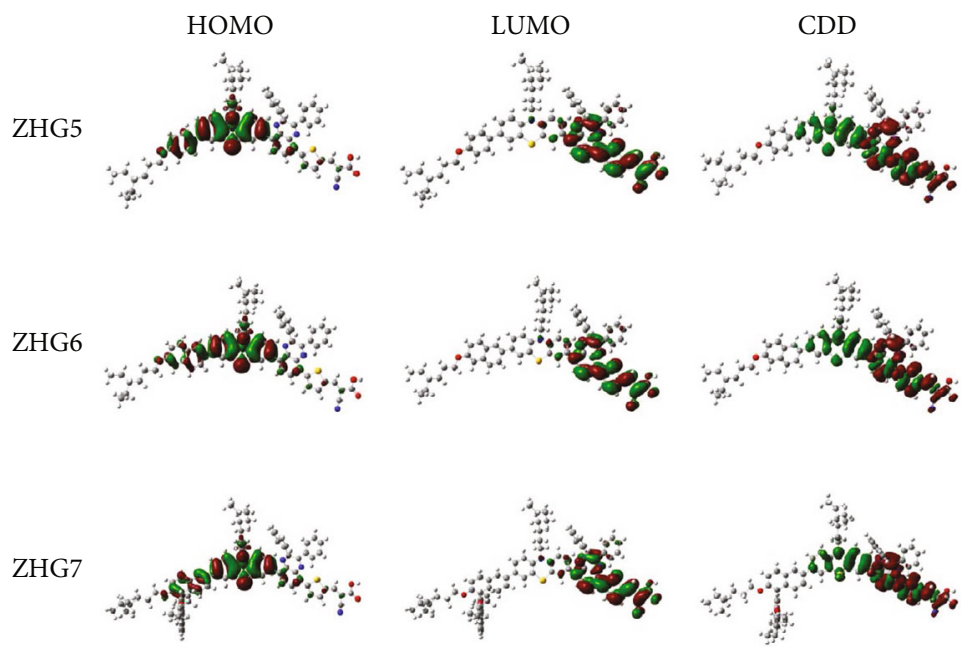

FIgURE 3: The molecular orbitals HOMO (left), LUMO (middle), and charge density difference (right) between the excited- and ground-state diagrams of three original molecules.

TABLE 3: Ionization potentials (IP), electron affinities (EA), electronegativity $(\chi)$, hardness $(\eta)$, softness $\left(S / \mathrm{eV}^{-1}\right)$, chemical potential $(\mu)$, electrophilicity index $(\Delta \mathrm{E})$, and total molecular energies ( $E_{\text {TOTAL }} /$ Hartree) of ZHG5, ZHG6, and ZHG7 (eV).

\begin{tabular}{lccc}
\hline & ZHG5 & ZHG6 & ZHG7 \\
\hline IP & 4.813 & 4.826 & 4.827 \\
EA & 2.995 & 2.996 & 2.995 \\
$\chi$ & 6.311 & 6.324 & 6.325 \\
$\eta$ & 3.316 & 3.328 & 3.330 \\
$S$ & 0.726 & 0.725 & 0.725 \\
$\mu$ & -3.904 & -3.911 & -3.911 \\
$\Delta \mathrm{E}$ & -1.818 & -1.830 & -1.832 \\
$E_{\text {TOTAL }}$ & -3639.847 & -3793.493 & -4567.906 \\
\hline
\end{tabular}

$\left(\lambda_{\text {abs }}\right)$ and maximum oscillator strengths $(f)$ were listed in Table 4 and Table S2. In addition, the absorption spectra of the three molecules are displayed in Figure 4. For ZHG5, the $\lambda_{\text {abs }}$ and $f$ of the first excited state are $452.36 \mathrm{~nm}$ and 1.450. Compared with ZHG5, the $\lambda_{\mathrm{abs}}$ simulated by ZHG6 is $451.50 \mathrm{~nm}$ and an oscillator of 1.499. In addition, the $\lambda_{\mathrm{abs}}$ of ZHG7 is $452.00 \mathrm{~nm}$ with the oscillator of 1.516 . The simulated UV-Vis absorption spectrum curves agree with the experimental data, and it can be seen that the addition of auxiliary donor does not change the absorption property of the sensitizer.

The fluorescence performance of the sensitizer can reflect whether the sensitizer can be used as the photosensitive layer of DSSC. When the fluorescence lifetime of the molecule is long, the excited state of the electron lasts for a long time, which is conducive to electron transfer. The emission characteristics are affected by many factors, such as the molar extinction coefficient, the vertical emission energy, and the simulated data are summarized in Table 5 . The wavelengths of the wavelength of fluorescence emission peak $\left(\lambda_{\mathrm{em}}\right)$ are higher $(\sim 120 \mathrm{~nm})$ than those of the wavelengths of the maximum molar extinction coefficient $\left(\lambda_{\mathrm{abs}}\right)$ for the three dyes.
Moreover, the fluorescence lifetime of the sensitizers can be derived by the following formula [46]:

$$
\tau=\frac{c^{3}}{2\left(E_{\mathrm{flu}}\right)^{2} f},
$$

here, $f$ represents the oscillator of the fluorescence spectrum. $c$ stands for the velocity of light and $E_{\text {flu }}$ means the vertical emission energy. $\tau$ is the fluorescence lifetime. The $\tau$ of the three sensitizers in S1 state is shown in Table 5, and it can be seen that the fluorescence lifetime of the sensitizers decreases with the increase of the auxiliary donor group. The specific relationship between molecules is ZHG5 (3.00 ns) > ZHG6 $(2.93 \mathrm{~ns})>$ ZHG7 $(2.91 \mathrm{~ns})$, which indicates that sensitizer ZHG5 is more suitable to be the photosensitive layer of DSSC than the other two sensitizers.

3.5. External Electric Field Analysis. Electron transfer is affected by the electric field, and the appropriate direction and intensity of the electric field can enhance the electron transfer of the sensitizer to the semiconductor, so it is particularly necessary to simulate the optical properties of sensitizers in the electric field. The external electric field of different strengths $\left(F=0,5 \times 10^{-4}, 10 \times 10^{-4}, 15 \times 10^{-4}\right.$, $20 \times 10^{-4}, 25 \times 10^{-4}$, and $30 \times 10^{-4}$ au) along the $x$-axis (The direction of the donor to the acceptor) was applied to the three sensitizers. Since the oxygen-containing group can be easily anchored to the semiconductor substrate, the direction of the external electric field is perpendicular to the semiconductor substrate $[47,48]$. The energy levels and gaps of the three sensitizers in the applied electric field of different intensities are summarized in Table S3. Comparing with the HOMO energy level without the intensity of the external electric field $(-4.94 \mathrm{eV}$ for ZHG5), it can be found that when the applied external electric field intensity is between 0 and $15 \times 10^{-4} \mathrm{au}$, the energy level increases with the increase of the external electric field. In addition, the LUMO level and energy gap decrease as the 
TABLE 4: The calculated transition properties of ZHG5, ZHG6, and ZHG7 in THF via TD-DFT/Cam-B3LYP/6-31G(d).

\begin{tabular}{|c|c|c|c|c|c|c|}
\hline Dyes & State & $E(\mathrm{eV})$ & $\lambda_{\text {abs }}(\mathrm{nm})$ & Contribution $\mathrm{MO}$ & Strength $(f)$ & Experiment (nm) \\
\hline \multirow{3}{*}{ ZHG5 } & S1 & 2.74 & 452.36 & $(51.7 \%) \mathrm{H} \longrightarrow \mathrm{L}$ & 1.450 & \multirow{3}{*}{471} \\
\hline & S2 & 3.28 & 377.97 & $(34.4 \%) \mathrm{H}-1 \longrightarrow \mathrm{L}$ & 0.198 & \\
\hline & S3 & 3.60 & 335.51 & $(14.9 \%) \mathrm{H}-8 \longrightarrow \mathrm{L}$ & 0.290 & \\
\hline \multirow{3}{*}{ ZHG6 } & S1 & 2.75 & 451.50 & $(49.6 \%) \mathrm{H} \longrightarrow \mathrm{L}$ & 1.499 & \multirow{3}{*}{466} \\
\hline & S2 & 3.28 & 377.44 & $(35.3 \%) \mathrm{H}-2 \longrightarrow \mathrm{L}$ & 0.178 & \\
\hline & S3 & 3.70 & 335.44 & $(21.9 \%) \mathrm{H}-9 \longrightarrow \mathrm{L}$ & 0.054 & \\
\hline \multirow{3}{*}{ ZHG7 } & S1 & 2.74 & 452.00 & $(49.0 \%) \mathrm{H} \longrightarrow \mathrm{L}$ & 1.516 & \multirow{3}{*}{472} \\
\hline & S2 & 3.29 & 377.16 & $(41.0 \%) \mathrm{H}-3 \longrightarrow \mathrm{L}$ & 0.182 & \\
\hline & S3 & 3.70 & 335.53 & $(11.5 \%) \mathrm{H}-11 \longrightarrow \mathrm{L}$ & 0.051 & \\
\hline
\end{tabular}

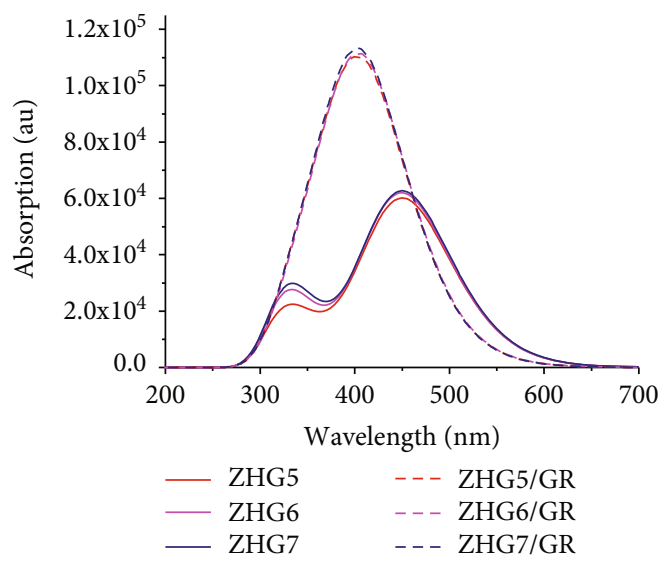

FIgURE 4: The UV-Vis absorption spectra of three isolated molecules and Dye/GR complexes in solvent.

TABLE 5: Emission properties of dyes ZHG5, ZHG6, and ZHG7.

\begin{tabular}{lccccc}
\hline Dye & State & $\begin{array}{c}E_{\text {flu }}(\mathrm{eV}) / \lambda_{\mathrm{em}} \\
(\mathrm{nm})\end{array}$ & $f$ & $\tau_{1}(\mathrm{~ns})$ & $\begin{array}{c}\text { Contribution } \\
\mathrm{MO}\end{array}$ \\
\hline ZHG5 & $\mathrm{S} 1$ & $2.18 / 569$ & 1.6163 & 3.00 & $\mathrm{H} \longrightarrow \mathrm{L} / 76.7 \%$ \\
ZHG6 & $\mathrm{S} 1$ & $2.18 / 568$ & 1.6565 & 2.93 & $\mathrm{H} \longrightarrow \mathrm{L} / 76.1 \%$ \\
ZHG7 & $\mathrm{S} 1$ & $2.18 / 568$ & 1.6674 & 2.91 & $\mathrm{H} \longrightarrow \mathrm{L} / 75.8 \%$ \\
\hline
\end{tabular}

external electric field increases. Not only that, when the electric field intensity is greater than $15 \times 10^{-4}$, there is no regularity in the change of the molecular energy level. For ZHG6, when the external electric field strength is $5 \times 10^{-4}$ $\mathrm{au}$, the HOMO value is very small and resulting in a large energy gap. Besides, the LUMO and energy gap all decrease with the increase of the $F$, and the HOMO decreases as the $F$ increases. When the $F$ is greater than $20 \times 10^{-4}$ au, the $\mathrm{HOMO}$ is greater than $-4.6 \mathrm{eV}$, indicating that the $F$ is appropriate between 0 and $20 \times 10^{-4}$ au. In addition, it is worth noting that the HOMO energy level can be adjusted by controlling the strength of the applied $F$, so as to affect whether DSSC works properly. According to this principle, the sensitizer can be designed as a nonlinear optical switch controlled by the electric field. For ZHG7, the variation trend of energy gap and energy level in the external electric field is the same as that of ZHG5.
The optical properties of the three sensitizers in electric fields of different intensities are shown in Figure 5 and Table S4. For three molecules, the absorption peak red shifts as the strength of the external electric field increases, and the oscillator strength of S1 decreases as the external electric field increases. By means of the absorption spectrum, it can be found that the absorption interval of dye in the visible region increases with the increase of the external electric field intensity, which is favorable for the sensitizer to be excited by light.

3.6. Molecular Electrostatic Potential (MEP). In order to examine the differential mutagenicities among three dyes, the molecular electrostatic potential (MEP) was calculated, and the results are shown in Figure 6. The MEP maps can directly reflect the reaction area of nucleophilic attack and electrophilic attack on molecules, and the different colors at the surface represent the different values of MEP. The electrostatic potential is in increasing order red $<$ pink $<$ buff $<$ palegreen $<$ mazarine. The color gradient of electrostatic potential ranges from $-0.06 \mathrm{au}$ (red) to $0.06 \mathrm{au}$ (mazarine). The MEP maps of all sensitizers showed that the hydrogen atom with hydroxyl groups in the acceptor had the highest positive potential compared with other parts of the molecule, indicating the preferred site for nucleophilic attack. The relatively negative potential regions are observed on nitrogen atom with the cyano group and oxygen atom with the carbonyl group in the acceptor part, which is the preferred area of electrophilic attack. It can be seen from the MEP maps that both nucleophilic attack and electrophilic attack are concentrated in the acceptor part of the sensitizers, indicating that the acceptor parts are the unstable region for three dyes. Therefore, the graphene quantum dot can be inserted in the acceptor parts to simulate the optical properties of the doped graphene on the device.

3.7. Properties of Dye/Graphene (Dye/GR) Complex. In order to prevent the $\mathrm{sp}^{2}$ hybridization network structure of graphene from being destroyed by the chemical functionalization of graphene oxide, inhibition of graphene oxidation has been applied to the preparation of Dye/GR nanomaterials. The cir-coronene graphene with $\mathrm{D}_{6 \mathrm{~h}}$ planar reticular structure was adopted as the quantum dot for anchoring sensitizers. The C-C single bond is formed between the sensitizer 


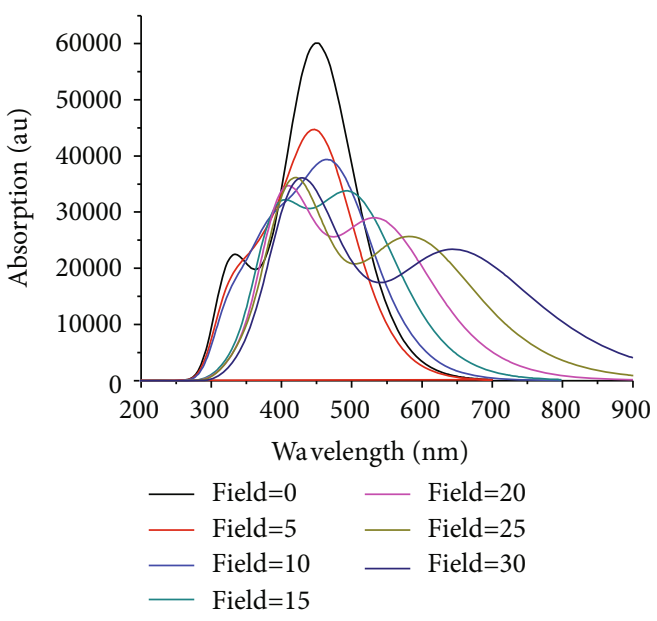

(a)

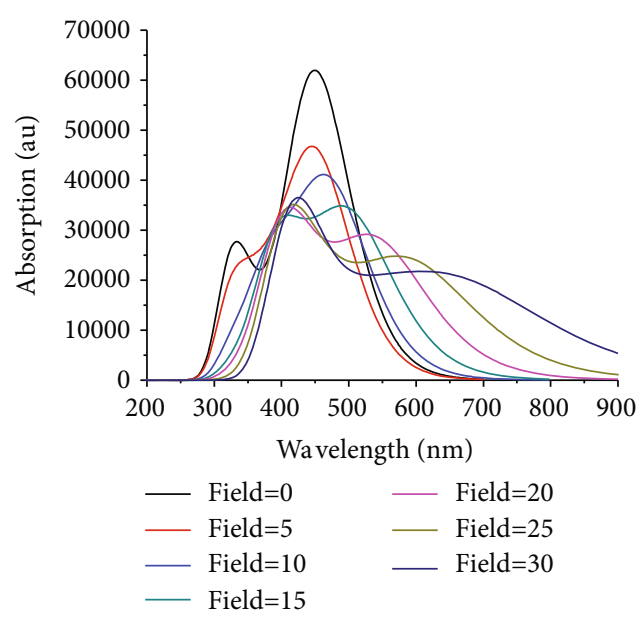

(b)

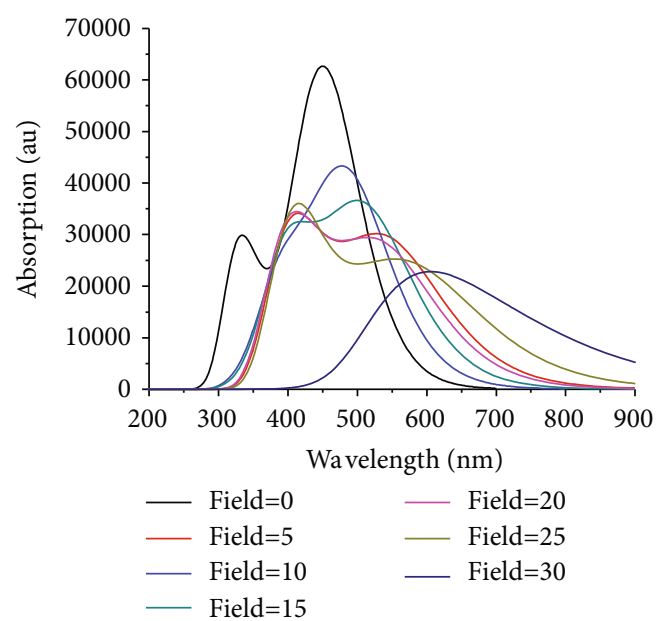

(c)

Figure 5: Simulated absorption spectra of three molecules under different electric field intensities ( $\times 10^{-4}$ au): (a) ZHG5, (b) ZHG6, (c) ZHG7.

molecules and quantum dots through dehydration and condensation. The C-C bonds formed between the three sensitizers and the quantum dots range from 1.498 to $1.499 \AA$. This is a stable state compared to the length of the C-C bonds in the molecule (1.39-1.55 $\AA$ ).

The energy levels and energy gaps of Dye/GR are given in Table 2. As can be seen from the chart, the HOMO level of the complex did not change significantly after the sensitizer was hybrid with graphene quantum dots, but the LUMO levels of the three complexes decreased to different degrees, which leads to a smaller gap, and which facilitates the transition of the molecular orbital.

The frontier molecular orbitals and optical properties are plotted in Figure S1, Figure 4 and Table S5. Table S5 shows that the oscillator intensity reaches its maximum when the molecular orbitals are transferred from HOMO-3 to LUMO, indicating that S2 is analyzed in detail. For ZHG5/GR, the maximum absorption peak in S2 state is $470.85 \mathrm{~nm}$, which is red shifted about $18 \mathrm{~nm}$ compared with the isolated sensitizer. Moreover, the oscillator strength of the sensitizer doped with graphene quantum dots also rises to 2.248, which is very beneficial to the light capture efficiency. In the excited-state S2, electrons are photoexcited from HOMO-3 to LUMO. On HOMO-3, the electron densities are distributed over the entire molecule, except for the second donor and graphene quantum dot. Moreover, the electron densities are occupied in the acceptor moiety of complex in the LUMO orbital.

The $\lambda_{\text {abs }}$ of the S2 for ZHG6/GR is $470.90 \mathrm{~nm}$, and the $f$ is 2.295. Compared with molecule ZHG6, the absorption peak of ZHG6/GR was red shifted about $19 \mathrm{~nm}$. This excited-state electron has a transition from HOMO-2 to LUMO. In HOMO-2, the electron densities are distributed on graphene quantum dots. Besides, the electron densities are distributed among the acceptor part of the complex in LUMO. For ZHG7/GR, the $\lambda_{\text {abs }}$ in S2 is $469.95 \mathrm{~nm}$, and the $f$ is 2.288, with the electron having a transition from HOMO-2 orbital to LUMO orbital in this state. For ZHG7, the $\lambda_{\text {abs }}$ of ZHG7/GR was red shifted about $17.95 \mathrm{~nm}$. In this excited state, the transition of electron density is similar to that of complex ZHG6/GR. As can be seen from the optical properties of the three complexes, the energy gaps of the complexes were significantly reduced after the graphene quantum dots were doped. At the same time, the molar 

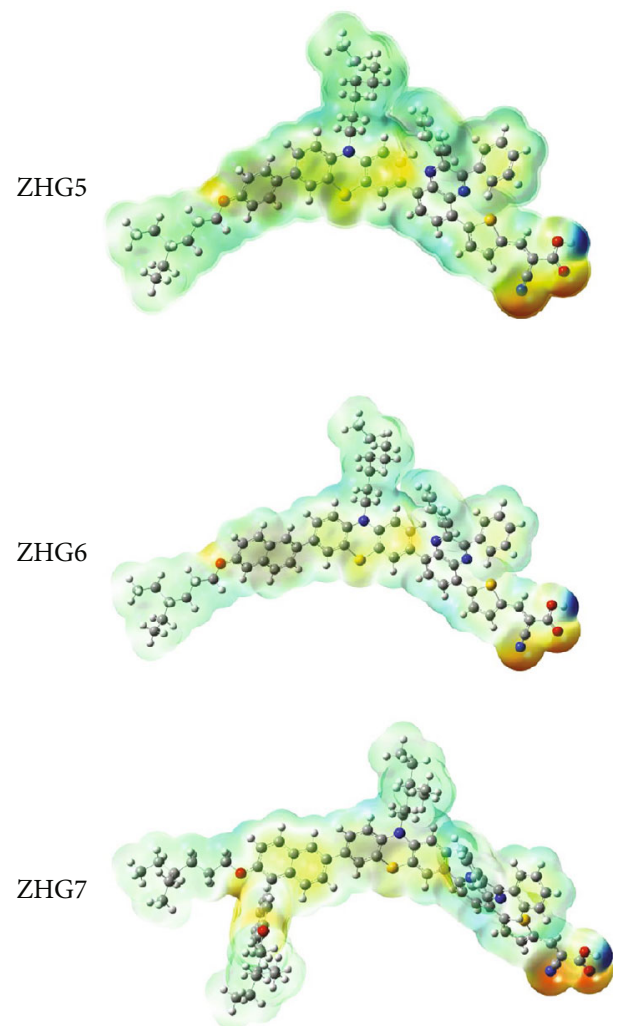

Figure 6: Molecular electrostatic potential plots of dyes ZHG5, ZHG6, and ZHG7 under uniform standards, where the red color depicts negative (electron-rich) regions (the preferred areas of electrophilic attack) and blue color depicts positive (electron-poor) regions (which preferred areas of nucleophilic attack).

extinction coefficients were also significantly increased, and the absorption spectrums were also significantly red shift. The simulation results evidenced that graphene quantum dot filling system compared with an isolated sensitizer is more advantageous to the optical performance of DSSCs.

3.8. Designed Molecules. The molar extinction coefficient, energy gap, fluorescence lifetime, and global reactivity descriptors of ZHG5 are better than those of ZHG6 and ZHG7. Based on the optimized spatial structure of ZHG5, nine sensitizers were designed by insertion of different functional groups (-OH, $-\mathrm{NH}_{2},-\mathrm{OCH}_{3},-\mathrm{CF}_{3},-\mathrm{F}$, and $\left.-\mathrm{CN}\right)$. The structural formulas for the nine design sensitizers are presented in Figure 7, where the $-\mathrm{OH},-\mathrm{NH}_{2}$, and $-\mathrm{OCH}_{3}$ groups are inserted into the donor portion, and the $-\mathrm{CF}_{3},-\mathrm{F}$, and $-\mathrm{CN}$ groups are inserted into the acceptor section.

The energy gaps and FMOs of the nine designed dye sensitizers are shown in Figure 2 and Figure S2, and the detailed data are summarized in Table S6. The HOMO energy level of the nine designed dyes is generally higher than that of the original molecule ZHG5, and the LUMO energy level decreased obviously (see Table 2). Therefore, compared with ZHG5, the energy gap of the nine designed molecules is reduced, in which ZHG5-3, ZHG5-6, and ZHG5-9 are decreased significantly. The optical properties of nine designed dyes are shown in Figure S3 and Table S7.

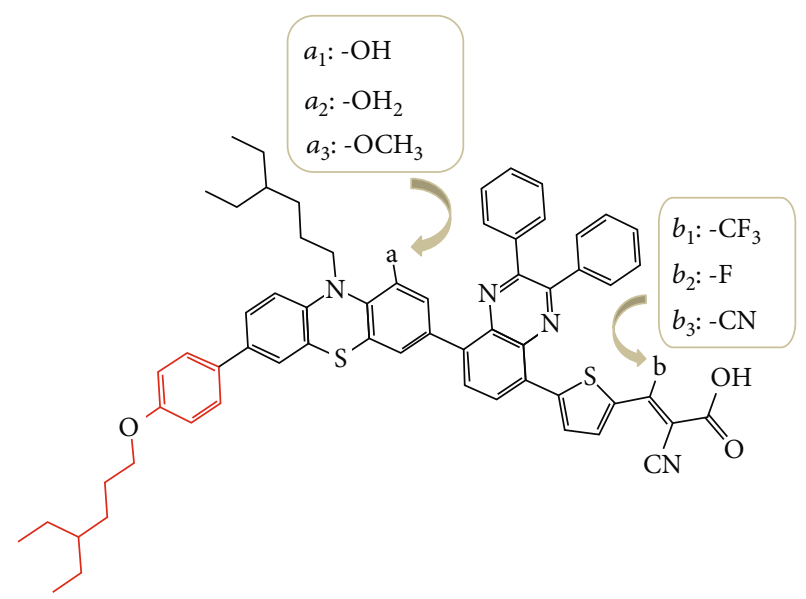

Figure 7: Chemical structures of the nine designed molecules, where ZHG5 $-1=\mathrm{ZHG} 5+a_{1}+b_{1}$, ZHG5 $-2=\mathrm{ZHG} 5+a_{1}+b_{2}$, ZHG5 $-3=\mathrm{ZHG} 5+a_{1}+b_{3}$, ZHG5 $-4=\mathrm{ZHG} 5+a_{2}+b_{1}$, ZHG5 $-5=$ ZHG5 $+a_{2}+b_{2}$, ZHG5 $-6=$ ZHG5 $+a_{2}+b_{3}$, ZHG5 $-7=$ $\mathrm{ZHG} 5+a_{3}+b_{1}$, ZHG5 $-8=\mathrm{ZHG} 5+a_{3}+b_{2}$, and ZHG5 $-9=$ $\mathrm{ZHG} 5+a_{3}+b_{3}$.

Compared to molecular ZHG5, the molar extinction coefficients of the designed molecules are generally reduced, and the absorption spectra of ZHG5-3, ZHG5-6, and ZHG5-9 show an obvious red shift.

In addition, in order to make the simulation results of the optical properties of the designed molecules more accurate, the sensitizers are anchored to the semiconductor and the properties of Dye/TiO 2 complexes are analyzed $[49,50]$. The sensitizer molecules and $\mathrm{TiO}_{2}$ are linked by a Ti-O single bond. The energy gaps and optical parameters of the nine complexes and ZHG5 are summarized in Table 6 and Table S6. The FMOs and absorption spectrum are available in Figures 8 and 9. By means of semiconductor anchoring, the simulation results can be more reliable and convincing. Therefore, the simulation results that the optical properties of ZHG5-3, ZHG5-6 and ZHG5-9 were better than the other six design molecules were verified again, and the optical properties of the sensitizers can be improved by inserting -CN into the acceptor part of the molecule ZHG5.

The adiabatic interface electron transfer theory is used to simulate the electron transfer from the sensitizer to the semiconducting band. By using the developed Newns-Anderson approach [51-54], the interfacial electron transfer (IET) time $\left(\tau_{2}\right)$ from sensitizers to a semiconductor can be simulated, and the detailed data are listed in Table 6. From Table 6, it can be found that the interfacial electron injection time of the designed molecules are in the same order of femtosecond.

\section{Conclusion}

In summary, the effects of steric hindrance and graphene quantum dots on the optical properties of DSSC were simulated by theoretical calculation. The results indicate that the donor part of ZHG5 was inserted into benzene ring and 2(4-ethylhexyloxy)naphthalene as space steric hindrance caused the energy gap and ionization energy were increased, 
TABLE 6: Calculated optical properties and interfacial electron-transfer time $\tau_{2}$ (fs) of the $\mathrm{ZHG} / \mathrm{TiO}_{2}$ and $\mathrm{Dye}_{\mathrm{TiO}}$ complexes on $\mathrm{S} 1$ state.

\begin{tabular}{|c|c|c|c|c|c|}
\hline Dyes & $E(\mathrm{eV})$ & $\lambda_{\text {abs }}(\mathrm{nm})$ & Contribution $\mathrm{MO}$ & Strength $(f)$ & $\tau_{2}$ \\
\hline $\mathrm{ZHG} 5 / \mathrm{TiO}_{2}$ & 2.59 & 478.48 & $(37.65 \%) \mathrm{H} \longrightarrow \mathrm{L}$ & 1.740 & 5.2 \\
\hline ZHG5-1/TiO 2 & 2.70 & 459.58 & $(35.10 \%) \mathrm{H} \longrightarrow \mathrm{L}+1$ & 1.300 & 5.1 \\
\hline ZHG5-2/ $\mathrm{TiO}_{2}$ & 2.60 & 478.20 & $(47.76 \%) \mathrm{H} \longrightarrow \mathrm{L}$ & 1.613 & 4.7 \\
\hline ZHG5-3/ $\mathrm{TiO}_{2}$ & 2.25 & 551.13 & $(51.73 \%) \mathrm{H} \longrightarrow \mathrm{L}$ & 1.543 & 6.3 \\
\hline ZHG5-4/TiO 2 & 2.56 & 484.71 & $(25.30 \%) \mathrm{H} \longrightarrow \mathrm{L}$ & 1.338 & 4.4 \\
\hline ZHG5-5/ $\mathrm{TiO}_{2}$ & 2.56 & 484.11 & $(50.21 \%) \mathrm{H} \longrightarrow \mathrm{L}$ & 1.532 & 4.7 \\
\hline ZHG5-6/ $\mathrm{TiO}_{2}$ & 2.21 & 559.92 & $(55.41 \%) \mathrm{H} \longrightarrow \mathrm{L}$ & 1.480 & 5.5 \\
\hline ZHG5-7/ $\mathrm{TiO}_{2}$ & 2.54 & 487.58 & $(37.13 \%) \mathrm{H} \longrightarrow \mathrm{L}+1$ & 1.367 & 4.4 \\
\hline ZHG5-8/ $\mathrm{TiO}_{2}$ & 2.59 & 479.27 & $(48.80 \%) \mathrm{H} \longrightarrow \mathrm{L}$ & 1.581 & 4.6 \\
\hline ZHG5-9/TiO 2 & 2.25 & 552.03 & $(52.70 \%) \mathrm{H} \longrightarrow \mathrm{L}$ & 1.524 & 5.7 \\
\hline
\end{tabular}

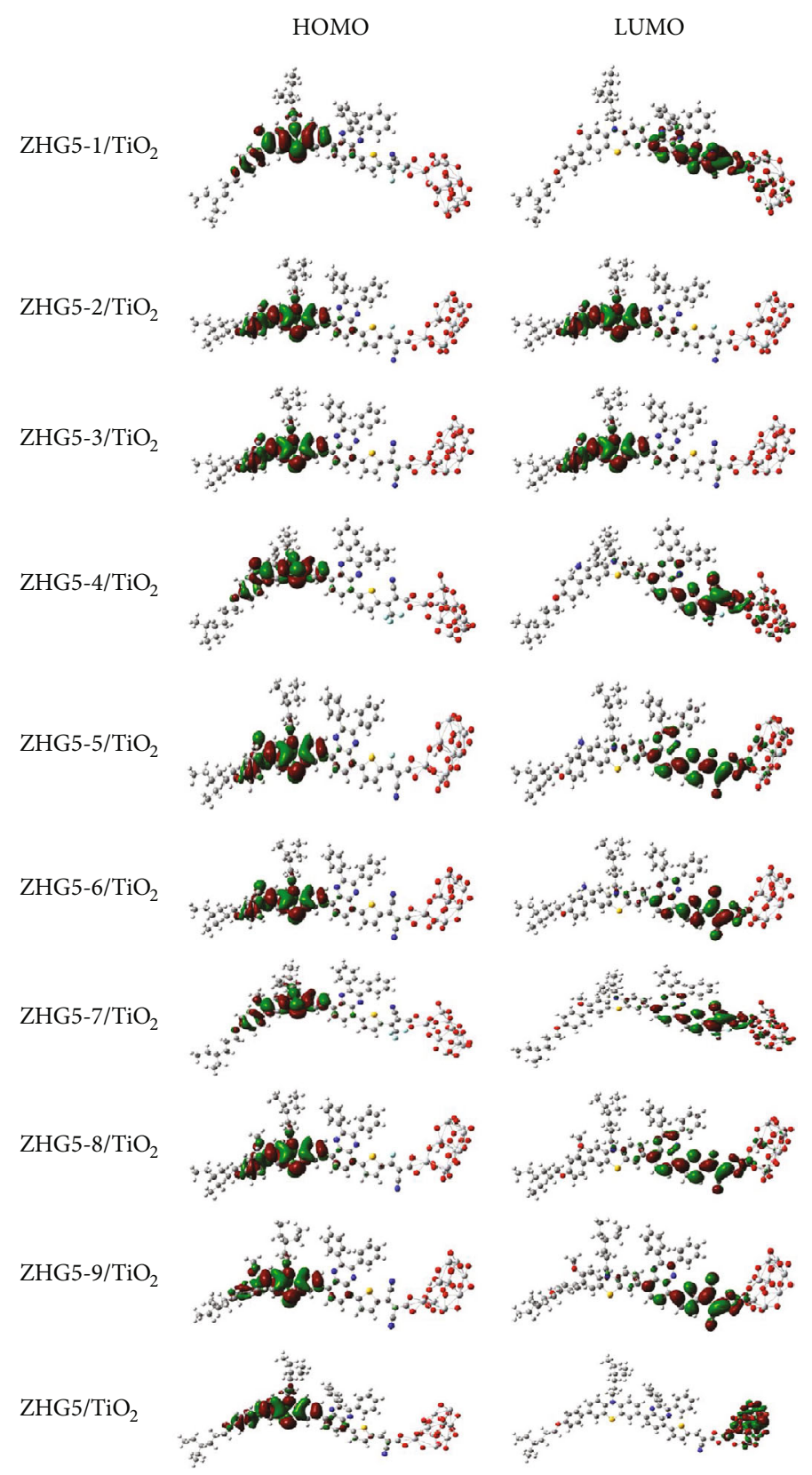

FIGURE 8: Selected frontier molecular orbitals of the designed Dye/ $/ \mathrm{TiO}_{2}$ and $\mathrm{ZHG} / \mathrm{TiO}{ }_{2}$ complexes in solvent. 


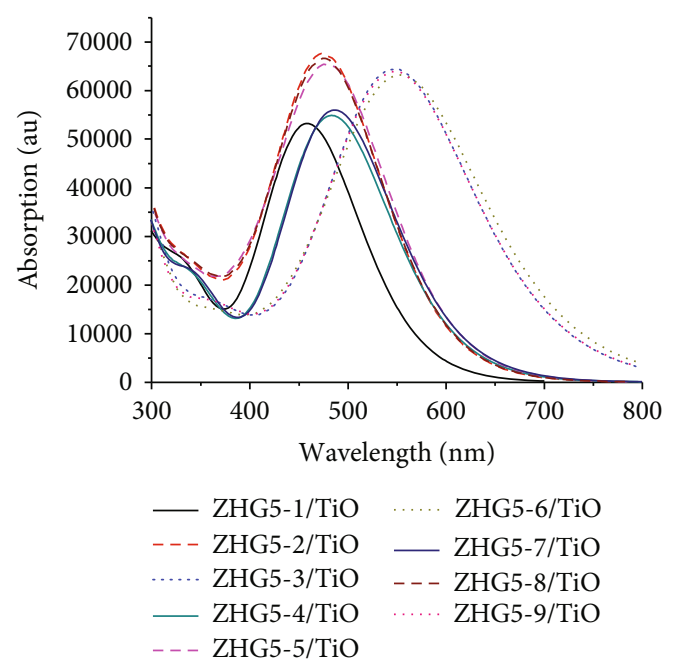

Figure 9: Simulated UV-Vis spectra of the designed Dye/TiO complexes in solvent.

and the fluorescence lifetime was reduced, which was not conducive to electron transfer and excited-state duration. This indicates that the steric hindrance effect is caused by the excessive size of the donor part, which is unfavorable to the overall performance of the sensitizer. Based on the analysis of the molecular electrostatic potential, a graphene quantum dot is anchored in the acceptor part with high electron activity, and it is found that the energy gaps of the Dye/GR complexes are reduced and the molar extinction coefficients are generally increased, which indicates that the sensitizer can improve the photoelectric properties of the device after anchoring graphene. The nine molecules were designed by inserting functional groups based on the molecular structure of ZHG5. The results showed that the improvement to the photoelectric properties of the molecules was the most obvious when - $\mathrm{CN}$ was inserted into the acceptor of the sensitizer. In addition, molecular dynamics of IET of the designed molecules were simulated, and the results showed that the dynamic transfer process of the designed molecules reached the femtosecond level, which was beneficial to the electron injection efficiency and short-circuit current.

\section{Data Availability}

The data used to support the findings of this study are available from the corresponding author upon request.

\section{Conflicts of Interest}

The authors declare no conflict of interest.

\section{Acknowledgments}

This work was supported by the National Natural Science Foundation of China (Grant Nos. 11974152 and 11404055), the Fundamental Research Funds for the Heilongiiang Postdoctoral Grant (LBH-Z15002), Fundamental Research Funds for China Postdoctoral Science Foundation (2016M590270), the Fundamental Research Funds for the Central Universities (2572018BC24) and the Heilongjiang Provincial Youth Science Foundation (QC2013C006).

\section{Supplementary Materials}

Table S1: experimental absorption peak, calculated absorption peaks with corresponding oscillator strengths of the dye ZHG5 in dichloromethane by using different functionals via TD-DFT/6-31G(d) method (nm). Table S2: the calculated transition properties of ZHG5, ZHG6 and ZHG7 in THF via TD-DFT/Cam-B3LYP/6-31G(d). Table S3: energy levels and energy gaps of the dyes in different external electric field $(\times 10-4 \mathrm{au})$, in which $\mathrm{H}$ and $\mathrm{L}$ represent HOMO and LUMO, respectively $(\mathrm{eV})$. Table $\mathrm{S} 4$ : calculated transition energies and oscillator strengths of ZHG5, ZHG6, and ZHG7 in electric field $(\times 10-4 \mathrm{au})$, in which $\mathrm{H}$ and $\mathrm{L}$ represent HOMO and LUMO, respectively. Table S5: the calculated transition properties of ZHG5/GR, ZHG6/GR, and ZHG7/GR in THF via TD-DFT/Cam-B3LYP/6-31G(d). Table S6: the calculated transition properties of the nine designed dyes and ZHG5 in solvent $(\mathrm{eV})$. Table S7: the calculated transition properties of the nine designed molecules in solvent $(\mathrm{eV})$. Figure $\mathrm{S1}$ : the frontier molecular orbits and charge density difference (right) between the excited- and ground-state diagrams of ZHG5/GR, ZHG6/GR, and ZHG7/GR. Figure S2: FMO diagrams of the designed molecules. Figure S3: simulated UV-Vis spectra of ZHG5 and nine designed molecules in solvent: (a) ZHG5, ZHG5-1, ZHG5-2, and ZHG5-3; (b) ZHG5, ZHG5-4, ZHG5-5, and ZHG5-6; (c) ZHG5, ZHG5-7, ZHG5-8, and ZHG5-9. (Supplementary Materials)

\section{References}

[1] B. O'Regan and M. Grätzel, "A low-cost, high-efficiency solar cell based on dye-sensitized colloidal $\mathrm{TiO}_{2}$ films," Nature, vol. 353, no. 6346, pp. 737-740, 1991.

[2] G. di Carlo, A. O. Biroli, F. Tessore, S. Caramori, and M. Pizzotti, " $\beta$-Substituted $\mathrm{Zn}^{\mathrm{II}}$ porphyrins as dyes for DSSC: A possible approach to photovoltaic windows," Coordination Chemistry Reviews, vol. 358, pp. 153-177, 2018.

[3] J. D. Roy-Mayhew and I. A. Aksay, "Graphene materials and their use in dye-sensitized solar cells," Chemical Reviews, vol. 114, no. 12, pp. 6323-6348, 2014.

[4] M. Daniels, F. de Jong, K. Kennes et al., "Promising Molecules for Optoelectronic Applications: Synthesis of 5,10-Dihydrobenzo[a ]indolo[2,3-c ]carbazoles by Scholl Reaction of 1,2Bis(indol-2-yl)benzenes," European Journal of Organic Chemistry, vol. 2018, no. 34, pp. 4683-4688, 2018.

[5] X. D. Wang, C. H. Yao, F. Wang, and Z. D. Li, "Cellulose-based nanomaterials for energy applications," Small, vol. 13, no. 42, p. 1702240, 2017.

[6] J. Zhang, H. C. Zhu, R. L. Zhong, L. Wang, and Z. M. Su, "Promising heterocyclic anchoring groups with superior adsorption stability and improved IPCE for high-efficiency noncarboxyl dye sensitized solar cells: a theoretical study," Organic Electronics, vol. 54, pp. 104-113, 2018.

[7] X. L. Shi, Y. H. Yang, L. H. Wang, and Y. Z. Li, "Introducing asymmetry induced by benzene substitution in a rigid fused $\pi$ spacer of $\mathrm{D}-\pi-\mathrm{A}$-Type solar cells: a computational 
investigation," Journal of Physical Chemistry C, vol. 123, no. 7, pp. 4007-4021, 2019.

[8] X.-F. Zang, Z.-S. Huang, H.-L. Wu et al., "Molecular design of the diketopyrrolopyrrole-based dyes with varied donor units for efficient dye-sensitized solar cells," Journal of Power Sources, vol. 271, pp. 455-464, 2014.

[9] L. Xu, C. Aumaitre, Y. Kervella et al., "Increasing the efficiency of organic dye-sensitized solar cells over $10.3 \%$ using locally ordered inverse opal nanostructures in the photoelectrode," Advanced Functional Materials, vol. 28, no. 15, p. 1706291, 2018.

[10] L. Huang, P. Ma, G. Deng et al., "Novel electron-deficient quinoxalinedithienothiophene- and phenazinedithienothiophenebased photosensitizers: The effect of conjugation expansion on DSSC performance," Dyes and Pigments, vol. 159, pp. 107114, 2018.

[11] I. Duerto, E. Colom, J. M. Andrés-Castán et al., "DSSCs based on aniline derivatives functionalized with a tert -butyldimethylsilyl group and the effect of the $\pi$-spacer," Dyes and Pigments, vol. 148, pp. 61-71, 2018.

[12] C. Chitpakdee, S. Jungsuttiwong, T. Sudyoadsuk, V. Promarak, N. Kungwan, and S. Namuangruk, "Modulation of $\pi$-spacer of carbazole-carbazole based organic dyes toward high efficient dye-sensitized solar cells," Spectrochimica Acta Part A: Molecular and Biomolecular Spectroscopy, vol. 174, pp. 7-16, 2017.

[13] Y. Li, X. Li, J. Qi, and Y. Xu, "Tunable optoelectronic properties of D-A- $\pi$-A type dyes by altering auxiliary acceptor position and atomic electronegativity," Journal of Molecular Liquids, vol. 287, p. 110883, 2019.

[14] P. H. Ren, C. F. Sun, Y. Shi, P. Song, Y. H. Yang, and Y. Z. Li, "Global performance evaluation of solar cells using two models: from charge-transfer and recombination mechanisms to photoelectric properties," Journal of Materials Chemistry C, vol. 7, no. 7, pp. 1934-1947, 2019.

[15] M. Xie, F. Q. Bai, J. J. Wang, Y. Q. Zheng, and Z. Y. Lin, “Theoretical investigations on the unsymmetrical effect of $\beta$-link $\mathrm{Zn}$ porphyrin sensitizers on the performance for dye-sensitized solar cells," Physical Chemistry Chemical Physics, vol. 20, no. 5, pp. 3741-3751, 2018.

[16] X. Y. Jin, L. B. Sun, D. Y. Li, C. L. Wang, and F. Q. Bai, "Efficiency difference between furan- and thiophene-based $\mathrm{D}-\pi-$ A dyes in DSSCs explained by theoretical calculations," RSC Advances, vol. 8, no. 52, pp. 29917-29923, 2018.

[17] B. Mandal, S. Sarkar, and P. Sarkar, "Theoretical studies on understanding the feasibility of porphyrin-sensitized graphene quantum dot solar cell," The Journal of Physical Chemistry $C$, vol. 119, no. 6, pp. 3400-3407, 2015.

[18] B. Rajbanshi and P. Sarkar, "Optimizing the photovoltaic properties of CdTe quantum dot-porphyrin nanocomposites: a theoretical study," The Journal of Physical Chemistry C, vol. 120, no. 32, pp. 17878-17886, 2016.

[19] C. Dong, X. Li, P. Jin, W. Zhao, J. Chu, and J. Qi, "Intersubunit electron transfer (IET) in quantum dots/graphene complex: what features does IET endow the complex with?," The Journal of Physical Chemistry C, vol. 116, no. 29, pp. 15833-15838, 2012.

[20] F. Gao, C. L. Yang, M. S. Wang, X. G. Ma, and W. W. Liu, "Theoretical studies on the possible sensitizers of DSSC: Nanocomposites of graphene quantum dot hybrid phthalocyanine/tetrabenzoporphyrin/tetrabenzotriazaporphyrins/cis-tetrabenzodiazaporphyrins/tetrabenzomonoazaporphyrins and their
Cu-metallated macrocycles," Spectrochimica Acta Part A: Molecular and Biomolecular Spectroscopy, vol. 195, pp. 176183, 2018.

[21] P. Li, Z. Wang, C. Song, and H. Zhang, "Rigid fused $\pi$-spacers in $\mathrm{D}-\pi-\mathrm{a}$ type molecules for dye-sensitized solar cells: a computational investigation," Journal of Materials Chemistry C, vol. 5, no. 44, pp. 11454-11465, 2017.

[22] S.-G. Chen, H.-L. Jia, X.-H. Ju, and H.-G. Zheng, "The impact of adjusting auxiliary donors on the performance of dyesensitized solar cells based on phenothiazine D-D- $\pi$-A sensitizers," Dyes and Pigments, vol. 146, pp. 127-135, 2017.

[23] P. Hohenberg and W. Kohn, "Inhomogeneous electron gas," Physical Review, vol. 136, no. 3B, pp. B864-B871, 1964.

[24] W. Kohn and L. J. Sham, "Quantum density oscillations in an inhomogeneous electron gas," Physical Review, vol. 137, no. 6A, pp. A1697-A1705, 1965.

[25] E. K. U. Gross and W. Kohn, "Local density-functional theory of frequency-dependent linear response," Physical Review Letters, vol. 55, no. 26, pp. 2850-2852, 1985.

[26] M. E. Casida, C. Jamorski, K. C. Casida, and D. R. Salahub, "Molecular excitation energies to high-lying bound states from time-dependent density-functional response theory: characterization and correction of the time-dependent local density approximation ionization threshold," The Journal of Chemical Physics, vol. 108, no. 11, pp. 4439-4449, 1998.

[27] A. D. Becke, "Density-functional exchange-energy approximation with correct asymptotic behavior," Physical Review A, vol. 38, no. 6, pp. 3098-3100, 1988.

[28] A. D. Becke, "Density-functional thermochemistry. III. The role of exact exchange," The Journal of Chemical Physics, vol. 98, no. 7, pp. 5648-5652, 1993.

[29] C. Lee, W. Yang, and R. G. Parr, "Development of the ColleSalvetti correlation-energy formula into a functional of the electron density," Physical Review B Condensed Matter, vol. 37, no. 2, pp. 785-789, 1988.

[30] W. Li, F. Q. Bai, J. Chen, J. Wang, and H. X. Zhang, "Planar amine-based dye features the rigidified O-bridged dithiophene $\pi$-spacer: A potential high-efficiency sensitizer for dyesensitized solar cells application," Journal of Power Sources, vol. 275, pp. 207-216, 2015.

[31] T. Yanai, D. P. Tew, and N. C. Handy, "A new hybrid exchange-correlation functional using the Coulombattenuating method (CAM-B3LYP)," Chemical Physics Letters, vol. 393, no. 1-3, pp. 51-57, 2004.

[32] J. P. Perdew, K. Burke, and M. Ernzerhof, "Generalized gradient approximation made simple," Physical Review Letters, vol. 77, no. 18, pp. 3865-3868, 1996.

[33] Y. Tawada, T. Tsuneda, S. Yanagisawa, T. Yanai, and K. Hirao, "A long-range-corrected time-dependent density functional theory," The Journal of Chemical Physics, vol. 120, no. 18, pp. 8425-8433, 2004.

[34] O. A. Vydrov and G. E. Scuseria, "Assessment of a long-range corrected hybrid functional," The Journal of Chemical Physics, vol. 125, no. 23, p. 234109, 2006.

[35] J.-D. Chai and M. Head-Gordon, "Systematic optimization of long-range corrected hybrid density functionals," The Journal of Chemical Physics, vol. 128, no. 8, p. 084106, 2008.

[36] C. Adamo and V. Barone, "Exchange functionals with improved long-range behavior and adiabatic connection methods without adjustable parameters: the mPW and mPW1PW models," The Journal of Chemical Physics, vol. 108, no. 2, pp. 664-675, 1998. 
[37] V. Barone and M. Cossi, "Quantum calculation of molecular energies and energy gradients in solution by a conductor solvent model," The Journal of Physical Chemistry A, vol. 102, no. 11, pp. 1995-2001, 1998.

[38] D. M. Newns, "Self-consistent model of hydrogen Chemisorption," Physics Review, vol. 178, no. 3, pp. 1123-1135, 1969.

[39] R. A. Marcus and N. Sutin, "Electron transfers in chemistry and biology," Biochimica et Biophysica Acta, vol. 811, no. 3, pp. 265-322, 1985.

[40] M. J. Frisch, G. W. Trucks, H. B. Schlegel et al., Gaussian $\sim 09$ Revision D.01, Gaussian, Inc., Wallingford, CT, USA, 2009.

[41] M. Grätzel, "Photoelectrochemical cells," Nature, vol. 414, no. 6861, pp. 338-344, 2001.

[42] L.-J. He, W. Wei, J. Chen, R. Jia, J. Wang, and H.-X. Zhang, "The effect of D-[De- $\pi-A] n(n=1,2,3)$ type dyes on the overall performance of DSSCs: a theoretical investigation," Journal of Materials Chemistry C, vol. 5, no. 30, pp. 75107520, 2017.

[43] R. G. Pearson, "Absolute electronegativity and hardness: application to inorganic chemistry," Journal of Organic Chemistry, vol. 27, no. 4, pp. 734-740, 1988.

[44] Z. Z. Sun, Q. S. Li, P. P. Sun, and Z. S. Li, "Probing the regeneration process of triphenylamine-based organic dyes in dyesensitized solar cells," Journal of Power Sources, vol. 276, pp. 230-237, 2015.

[45] R. Gayathri, "An experimental and theoretical investigation of the electronic structure and photoelectrical properties of 1 , 4-diacetoxy-2-methylnaphthalene for DSSC application," Journal of Molecular Structure, vol. 1166, pp. 63-78, 2018.

[46] I. Litani-Barzilai, V. Bulatov, V. V. Gridin, and I. Schechter, "Detector based on time-resolved ion-induced voltage in laser multiphoton ionization and laser-induced fluorescence," Analytica Chimica Acta, vol. 501, no. 2, pp. 151156, 2004.

[47] K. E. Lee, M. A. Gomez, S. Elouatik, and G. P. Demopoulos, "Further understanding of the adsorption mechanism of N719 sensitizer on anatase TiO2 films for DSSC applications using vibrational spectroscopy and confocal Raman imaging," Langmuir, vol. 26, no. 12, pp. 9575-9583, 2010.

[48] H. C. Zhu, C. F. Li, Z. H. Fu, S. S. Wei, X. F. Zhu, and J. Zhang, "Increasing the open-circuit voltage and adsorption stability of squaraine dye binding onto the $\mathrm{TiO}_{2}$ anatase (llll $\left.\begin{array}{ll}0 & 1\end{array}\right)$ surface via heterocyclic anchoring groups used for DSSC," Applied Surface Science, vol. 455, pp. 1095-1105, 2018.

[49] A. Irfan, A. R. Chaudhry, S. Muhammad, and A. G. Al-Sehemi, "Exploring the potential of boron-doped nanographene as efficient charge transport and nonlinear optical material: a firstprinciples study," Journal of Molecular Graphics, vol. 75, pp. 209-219, 2017.

[50] S. H. Li, C. R. Zhang, L. H. Yuan et al., "The role of electronic donor moieties in porphyrin dye sensitizers for solar cells: electronic structures and excitation related properties," Journal of Renewable and Sustainable Energy, vol. 9, no. 5, 2017.

[51] J. P. Muscat and D. M. Newns, "Chemisorption on metals," Progress in Surface Science, vol. 9, no. 1, pp. 1-43, 1978.

[52] Y. M. Hailu, M. T. Nguyen, and J. C. Jiang, "Effects of the terminal donor unit in dyes with D-D- $\pi-A$ architecture on the regeneration mechanism in DSSCs: a computational study," Physical Chemistry Chemical Physics, vol. 20, no. 36, pp. 23564-23577, 2018.
[53] Y. C. Li, J. Y. Liu, D. X. Liu, X. Li, and Y. L. Xu, "D-A- $\pi-A$ based organic dyes for efficient DSSCs: A theoretical study on the role of $\pi$-spacer," Computational Materials Science, vol. 161, pp. 163-176, 2019.

[54] N. Wazzan and A. Irfan, "Theoretical study of triphenylaminebased organic dyes with mono-, di-, and tri-anchoring groups for dye-sensitized solar cells," Organic Electronics, vol. 63, pp. 328-342, 2018. 